$11-2012$

\title{
Ultra-thin perfect absorber employing a tunable phase change material
}

Mikhail A. Kats

Deepika Sharma

$\cdots$

M. M. Qazilbash

William \& Mary, mmqazilbash@wm.edu

et al.

Follow this and additional works at: https://scholarworks.wm.edu/aspubs

Part of the Condensed Matter Physics Commons

\section{Recommended Citation}

Kats, Mikhail A.; Sharma, Deepika; ...; Qazilbash, M. M.; and al., et, Ultra-thin perfect absorber employing a tunable phase change material (2012). Applied Physics Letters, 101(22).

https://doi.org/10.1063/1.4767646

This Article is brought to you for free and open access by the Arts and Sciences at W\&M ScholarWorks. It has been accepted for inclusion in Arts \& Sciences Articles by an authorized administrator of W\&M ScholarWorks. For more information, please contact scholarworks@wm.edu. 


\title{
Ultra-thin perfect absorber employing a tunable phase change material
}

\author{
Mikhail A. Kats, ${ }^{1}$ Deepika Sharma, ${ }^{1,2}$ Jiao Lin, ${ }^{1,3}$ Patrice Genevet, ${ }^{1}$ Romain Blanchard, ${ }^{1}$ \\ Zheng Yang, ${ }^{1}$ M. Mumtaz Qazilbash, ${ }^{4,5}$ D. N. Basov, ${ }^{4}$ Shriram Ramanathan, ${ }^{1}$ \\ and Federico Capasso ${ }^{1}$ \\ ${ }^{1}$ School of Engineering and Applied Sciences, Harvard University, Cambridge, Massachusetts 02138, USA \\ ${ }^{2}$ Department of Physics and Mathematics, University of Eastern Finland, Joensuu 80101, Finland \\ ${ }^{3}$ Singapore Institute of Manufacturing Technology, Singapore 638075, Singapore \\ ${ }^{4}$ Department of Physics, University of California-San Diego, La Jolla, California 92093, USA \\ ${ }^{5}$ Department of Physics, College of William and Mary, Williamsburg, Virginia 23187, USA
}

(Received 5 August 2012; accepted 4 September 2012; published online 26 November 2012)

\begin{abstract}
We show that perfect absorption can be achieved in a system comprising a single lossy dielectric layer of thickness much smaller than the incident wavelength on an opaque substrate by utilizing the nontrivial phase shifts at interfaces between lossy media. This design is implemented with an ultra-thin $(\sim \lambda / 65)$ vanadium dioxide $\left(\mathrm{VO}_{2}\right)$ layer on sapphire, temperature tuned in the vicinity of the $\mathrm{VO}_{2}$ insulator-tometal phase transition, leading to $99.75 \%$ absorption at $\lambda=11.6 \mu \mathrm{m}$. The structural simplicity and large tuning range (from $\sim 80 \%$ to $0.25 \%$ in reflectivity) are promising for thermal emitters, modulators, and bolometers. @ 2012 American Institute of Physics. [http://dx.doi.org/10.1063/1.4767646]
\end{abstract}

In optics, resonant optical cavity configurations have been used for detectors and modulators to achieve enhanced absorption at selected wavelengths and a high on-off ratio, respectively. The design of such devices benefits from critical coupling, a phenomenon which facilitates efficient power transfer to a resonator, which occurs when the internal losses of a resonant cavity are equal to the mirror losses, i.e., due to light escaping from the device facets. ${ }^{1}$ One implementation of this concept is the asymmetric Fabry-Perot (FP) cavity in which the dielectric forming the cavity is typically at least a quarter-wave in thickness and is surrounded by mirrors with unequal reflectivities, in which the back reflector is often a Bragg mirror. ${ }^{2-7}$ This geometry has been used for reflection modulators, ${ }^{2,5,6}$ for resonant-cavity enhanced (RCE) photodetectors, ${ }^{3,4}$ and for enabling strong coupling between light and matter. ${ }^{7}$ More recently, the concept of critical coupling has been reformulated in terms of the time reversal of lasing at threshold or "coherent perfect absorption." example of critically coupled resonators is the class of perfect absorbers comprising plasmonic nanostructures, which have been demonstrated over a wide range of frequencies, with typical experimental absorption values of $\sim 90 \%$ (Refs. 11-14) and reaching as high as $99 \% .{ }^{15}$ Unlike the asymmetric FPs, these nanostructured devices are very thin compared to the wavelength of light, but have complex nanofabrication requirements, which may limit practical device applications. It is sometimes assumed that perfect absorbers based on dielectric cavities cannot be made much thinner than the operating wavelength, and that plasmonic nanostructures are required to overcome this limitation. ${ }^{10}$

In this Letter, we demonstrate a perfect absorber comprising an unpatterned, ultra-thin $(\sim \lambda / 65)$ film of vanadium dioxide $\left(\mathrm{VO}_{2}\right)$ on an sapphire substrate. By utilizing an intermediate state of the insulator-metal phase transition (IMT) in $\mathrm{VO}_{2}$, which exhibits multiple co-existing phases, an effective medium with tunable optical properties is formed. In particular, the absorption coefficient can be very large in proximity to the IMT. We show that thermal control of the phase co-existence in the $\mathrm{VO}_{2}$ film enables switching of the absorption from $\sim 20 \%$ to $\sim 99.75 \%$ at $\lambda=11.6 \mu \mathrm{m}$. The absorption in our device is greatly enhanced via critical coupling to a cavity resonance, which is shown to exist when the ultra-thin film has a large imaginary part of the refractive index. Our device combines the deep-subwavelength thickness characteristic of nanostructure-based perfect absorbers with the wide tuning capability typical of asymmetric FP cavities, while comprising only a single film deposited on a reflecting substrate. This structural simplicity represents a significant advantage for the implementation of modulators, thermal emitters, and bolometers.

We consider light incident from air $\left(n_{1}=1\right)$ onto a dielectric film with complex refractive index $n_{2}+i k_{2}$, deposited on a substrate with index $n_{3}+i k_{3}$ (Fig. 1(a)). The reflection can be calculated as the coherent sum of the partial waves reflected from the first interface (with reflection coefficient $r_{0}$ ) and those reflected from the cavity after $1,2, \ldots, m$ roundtrips with reflection coefficients $r_{1}, r_{2}, \ldots, r_{m}$. We can then write the total reflectivity $R=|r|^{2}$, in terms of the reflection coefficient ${ }^{16}$

$$
r=\sum_{m=0}^{\infty} r_{m}=\frac{r_{12}+r_{23} e^{2 i \beta}}{1+r_{12} r_{23} e^{2 i \beta}},
$$

where $r_{p q}=\left(\tilde{n}_{p}-\tilde{n}_{q}\right) /\left(\tilde{n}_{p}+\tilde{n}_{q}\right)$ and $t_{p q}=2 \tilde{n}_{p} /\left(\tilde{n}_{p}+\tilde{n}_{q}\right)$ are the Fresnel reflection and transmission coefficients as the wave encounters medium $q$ from medium $p, \tilde{n}_{p}=n_{p}+i k_{p}$ is the complex refractive index of medium $p, \beta=\frac{2 \pi}{\lambda_{0}} \tilde{n}_{2} h$, $r_{0}=r_{12}$, and $r_{m}=t_{12} r_{23}{ }^{m} r_{21}{ }^{(m-1)} t_{21} e^{2 m i \beta}$ for $m>0$.

When $k_{2} \ll n_{2}$, Eq. (1) describes the reflection properties of a simple asymmetric FP cavity with small optical losses. On resonance, light is stored for many optical cycles and can be gradually absorbed as it circulates; most FP modulators and RCE detectors operate in this manner. Such a cavity is illustrated in Fig. 1(a), with a dielectric film deposited on a reflecting substrate. In the partial wave picture, the first reflection $r_{0}$ has a phase shift of $\simeq \pi$ with respect to the 

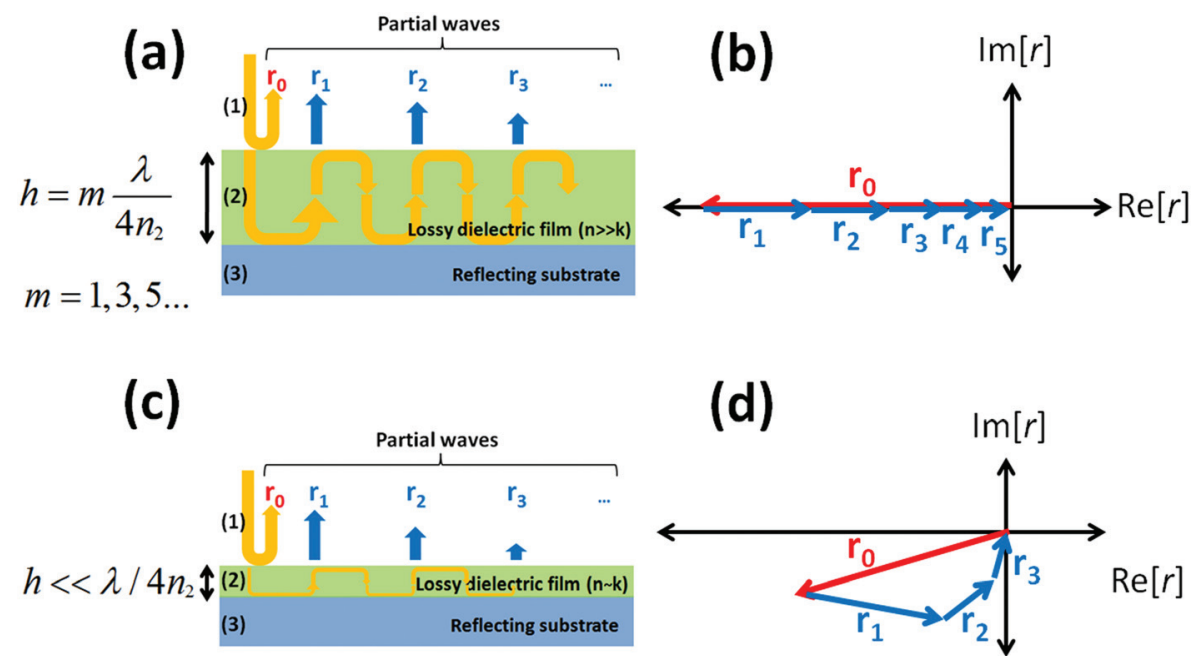

FIG. 1. (a) The reflection process from a quarter-wave film with low losses $\left(k_{2} \ll n_{2}\right)$ on a perfectly reflecting substrate at normal incidence, showing the partial waves. Many multiple reflections are involved because of the small losses. (b) Phasor addition diagram (the reflected partial waves are represented in the complex plane) demonstrating that a properly engineered quarter wave film on a reflecting substrate can result in zero reflection via destructive interference, corresponding to complete absorption. This occurs for a particular value of $k_{2}$, which is relatively small, leading to a small imaginary part of $r_{0}$, and corresponding to critical coupling. The phase of the first partial wave $r_{0}$ is $\simeq \pi$ with respect to the incident wave, but the phase of all of the other partial waves is $\simeq 0$. (c) Reflection process from a highly absorbing $\left(k_{2} \sim n_{2}\right.$ ), ultra-thin film in a reflecting substrate. (d) Phasor diagram demonstrating that a zero-reflection (and hence perfect absorption) condition is achievable if the complex refractive index of the film has a large imaginary component. In this case, the phase of $r_{0}$ deviates significantly from $\pi$ (the phasor is not along the horizontal axis) and a small number of reflections is sufficient to cancel $r_{0}$ and maximize absorption.

incident wave and thus the corresponding phasor points to the left, predominantly along the real axis in the complex plane (Fig. 1(b)). The front facet reflection can be cancelled out if the partial waves emerging from the film each have a phase shift of $\simeq 0$ and the phasor trajectory terminates at the origin; this occurs when the thickness $h$ of the dielectric is an odd integer multiple of $\lambda / 4 n$ (if the reflection phase from the substrate is $\pi$ ) and the reflectivity $\left|r_{12}\right|^{2}$ is equal to the effective bottom mirror reflectivity $\left|r_{23} e^{2 i \beta}\right|^{2}$, as can be seen from Eq. (1). ${ }^{5}$

The phasor diagram in Fig. 1(d) suggests another route to achieving the perfect absorption condition. The exact phasor trajectory does not matter as long as it returns to the origin. One of the possible trajectories in which the phase of $r_{0}$ is not $\pi$ is shown in Fig. 1(d). The interface reflection phase shifts become substantially different from 0 and $\pi$ when at least one of the materials has $k$ comparable to $n$. As a result, an absorption resonance can exist for a film that is much thinner than the wavelength of light, and critical coupling to this ultra-thin resonance yields a perfectly absorbing state (Fig. 1(c)). This effect has been recently utilized for the coloring of metals with nanometer-thick semiconductor films. ${ }^{40}$ Our perfect absorber utilizes this condition to enhance absorption, using the intermediate state in the IMT in $\mathrm{VO}_{2}$ to introduce a large, controllable degree of loss.

$\mathrm{VO}_{2}$ is a correlated oxide that undergoes a thermally triggered IMT near room temperature $\left(T_{c} \sim 340 \mathrm{~K}\right)$, which takes the material from an insulating state (band gap of $\sim 0.6 \mathrm{eV}$ ) to a metallic one. The IMT occurs gradually as the temperature is increased: nanoscale islands of the metallic phase emerge in the surrounding insulating $\mathrm{VO}_{2}$, which then grow and connect in a percolation process, leading to a fully metallic state where the band gap has collapsed. ${ }^{17-19}$ This metal-dielectric phase co-existence within the phase transition results in widely tunable optical properties; in fact, the naturally occurring nanoscale structures in the IMT region can be viewed as a reconfigurable disordered metamaterial. The IMT has been utilized for optical switching ${ }^{20,21}$ and has enabled several tunable devices comprising metallic nanostructures on $\mathrm{VO}_{2}$ films. ${ }^{22-24} \mathrm{VO}_{2}$ is also the target of active research for the realization of novel electronic switching devices that may complement MOSFET technology. ${ }^{19}$

To implement an absorber that can be tuned between low and perfect absorption states, we deposited a crystalline film of $\mathrm{VO}_{2}$ with a thickness of $180 \mathrm{~nm}$ on a c-plane sapphire substrate. The absorption was investigated via normal incidence measurements using a Fourier transform infrared (FTIR) spectrometer and mid-IR microscope (Fig. 2(a)). The reflection spectrum was collected while gradually increasing the sample temperature from $297 \mathrm{~K}$ to $360 \mathrm{~K}$ at $1 \mathrm{~K}$ increments (Fig. 2(b)). ${ }^{39}$ The $297 \mathrm{~K}$ curve is representative of the reflection spectrum at a temperature significantly below $T_{C}$. Since insulating $\mathrm{VO}_{2}$ is relatively transparent at photon energies below its band-gap, the primary features of the room temperature reflection spectrum are due to the underlying sapphire. Sapphire is highly absorptive at $\lambda \approx 5-10 \mu \mathrm{m}$ despite its large band gap due to the presence of several phonon modes, which also result in high reflectivity between 10 and $15 \mu \mathrm{m} .^{18,25}$ The $\mathrm{VO}_{2}$ thickness is much smaller than the wavelength of the incident light, so no FP fringes are observed. The small features at $\sim 3 \mu \mathrm{m}, \sim 4.5 \mu \mathrm{m}$, and $\sim 6 \mu \mathrm{m}$ correspond to ambient atmospheric absorption lines. At high temperatures (e.g., $360 \mathrm{~K}$ curve in Fig. 2(b)), the $\mathrm{VO}_{2}$ is entirely in the metallic phase and displays relatively high reflectivity, which slowly increases with increasing wavelength, as expected for a Drude-like metal.

The reflectivity spectrum does not transition monotonically from that of the low-temperature state to that of the hightemperature one due to the complex interplay between the effective medium formed when the $\mathrm{VO}_{2}$ is in an intermediate 

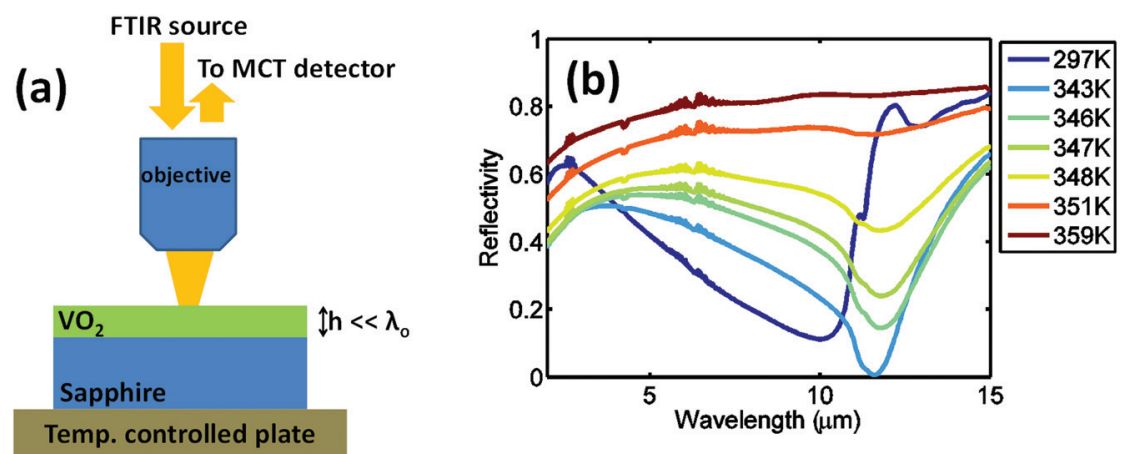

FIG. 2. (a) Experimental setup. A sapphire substrate coated with $h=180 \mathrm{~nm}$ of $\mathrm{VO}_{2}$ is placed on a temperature-controlled stage mounted inside an infrared (IR) microscope and illuminated at normal incidence using a mid-IR source. A mercury-cadmium-telluride (MCT) detector is used to collect the reflected light. (b) Experimental reflectivity spectrum at temperatures from $297 \mathrm{~K}$ to $360 \mathrm{~K}$. At $343 \mathrm{~K}$, the reflectivity drops to $\sim 0.0025$ at $\lambda=11.6 \mu \mathrm{m}$. (c) Experimental reflectivity from the sample at $\lambda=11.6 \mu \mathrm{m}$ as a function of increasing (red) and then decreasing (blue) temperature. $\mathrm{A} \sim 5 \mathrm{~K}$ hysteresis is seen in the reflectivity. Inset: Normalized $d c$ resistance of the $\mathrm{VO}_{2}$ thin film sample as a function of temperature showing nearly four orders of magnitude of change in the resistance and hysteretic behavior. (d) Calculated reflectivity spectrum at temperatures from $295 \mathrm{~K}$ to $360 \mathrm{~K}$ using experimental values for the complex refractive indices of $\mathrm{VO}_{2}$ (Ref. 17) and sapphire. ${ }^{26}$ The reflectivity of bare sapphire is shown in black.

state and the underlying sapphire substrate. In particular, we focus on the feature at $\lambda \sim 11.6 \mu \mathrm{m}$; at this wavelength, the reflectivity is $\sim 0.7$ with the $\mathrm{VO}_{2}$ in the insulating state (at room temperature) due to the high reflectivity of the underlying sapphire, and $\sim 0.8$ with the $\mathrm{VO}_{2}$ in the metallic state $(\mathrm{T}=360 \mathrm{~K})$. At $\mathrm{T}=343 \mathrm{~K}$, however, the reflectivity abruptly drops to approximately 0.0025 , corresponding to a reduction by a factor $\sim 280$ with respect to the low-temperature insulating state and by a factor $\sim 320$ with respect to the hightemperature metallic state. Since the sapphire substrate is opaque at this wavelength, this corresponds to a $99.75 \%$ absorbance within the $\mathrm{VO}_{2}$ film and the top part of the substrate (1$2 \mu \mathrm{m})$, as discussed later in the text. The reflectivity spectrum has a hysteresis of approximately $5 \mathrm{~K}$ (Fig. 2(c)), comparable to the $d c$ resistance hysteresis width of the $\mathrm{VO}_{2}$ film (inset of Fig. 2(c)). The normalized $d c$ resistance $R(T) / R(298 \mathrm{~K})$ exhibits a change of more than three orders of magnitude between $298 \mathrm{~K}$ and $393 \mathrm{~K}$.

To obtain the theoretical reflectivity of our device, we used Eq. (1) with the temperature-dependent complex refractive index for $\mathrm{VO}_{2}$ (for increasing temperature) experimentally obtained by ellipsometry in Ref. 17 and the index for sapphire from Ref. 26. The calculated spectra match well with the experimental data across the entire $\lambda=2-15 \mu \mathrm{m}$ range (Figs. 2(b) and 2(d)), though the temperatures at which the various spectral features occur differ by $2^{\circ}-5^{\circ}$. We attribute the latter to the differences in the growth conditions and film thicknesses between our $\mathrm{VO}_{2}$ sample and the one measured in Ref. $17 . .^{27}$ The predicted reflectivity minimum is $\sim 0.0007$ at $\lambda=11.75 \mu \mathrm{m}$ and $\mathrm{T} \sim 342 \mathrm{~K}$, compared to the experimental data, which showed a minimum value of $\sim 0.0025$ at $\lambda=11.6 \mu \mathrm{m}$ and $\mathrm{T}=343 \mathrm{~K}$.

To better understand the conditions leading to perfect absorption, we performed a set of calculations in which the $\mathrm{VO}_{2}$ film was replaced with an unknown homogeneous dielectric of the same thickness, assuming only that it can be described by some complex refractive index $\tilde{n}=n+i k$. In Fig. 3, we plotted the calculated reflectivity using Eq. (1) at $\lambda=11.75 \mu \mathrm{m}$ as a function of $n$ and $k$, covering a wide range of potential values of $\tilde{n}$ for the material comprising the thin film. The complex index of sapphire at this wavelength was taken to be $\sim 0.1+0.8 \mathrm{i}^{26}$ We found that for $\tilde{n} \cong 3.25+1.5 i$, the calculated reflectivity drops to zero indicating critical coupling, with $\sim 90 \%$ of the light absorbed in the $180 \mathrm{~nm}$ film and the remaining $\sim 10 \%$ absorbed in the top layer $(1-2 \mu \mathrm{m})$ of the underlying sapphire. This reflectivity minimum is very broad in $n-k$ space, making the phenomenon very robust; as a result, small changes in the composition (and hence $\tilde{n}$ ) of the lossy dielectric will not significant impact device

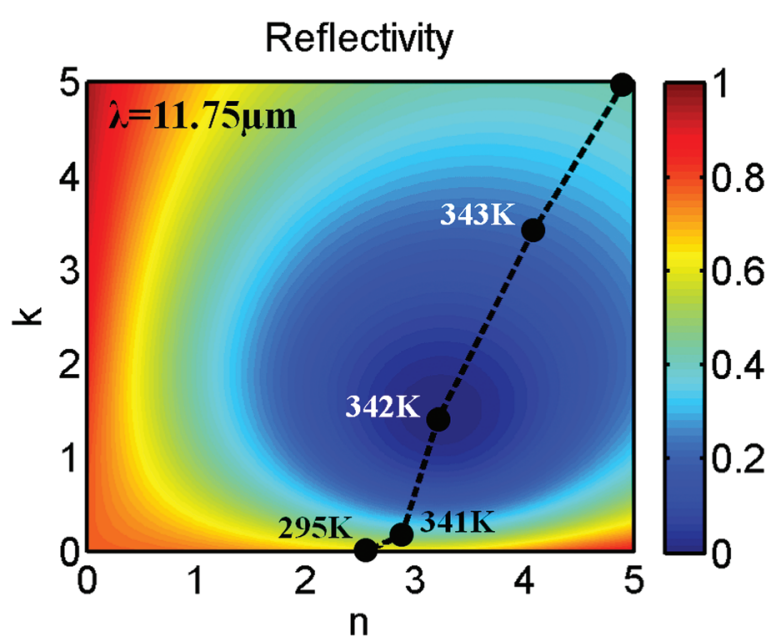

FIG. 3. (a) Map of the calculated reflectivity as a function of $\mathrm{n}$ and $\mathrm{k}$, the real and imaginary parts of the complex refractive index $\tilde{n}$, of a uniform dielectric film of $180 \mathrm{~nm}$ thickness on sapphire for $\lambda=11.75 \mu \mathrm{m}$. The reflectivity drops to zero for $\tilde{n} \cong 3.25+1.5 i$. The black dashed line marks the trajectory of the complex refractive index of $\mathrm{VO}_{2}$ with increasing temperature. The $\mathrm{VO}_{2}$ index passes very close to the minimum reflectivity point in $n-k$ parameter space (black dashed line). 
performance. The spectral width of the absorption minimum at the perfect absorption condition ( $\sim 3 \mu$ m full-width half-max) is determined primarily by the dispersion of the materials comprising the absorbing layer and the underlying substrate.

As shown in Figs. 1(c) and 1(d), complex values of the refractive indices lead to non-trivial reflection phase shifts (e.g., approximately $0.08 \pi$ for the $\mathrm{VO}_{2}$ /air interface and $-0.02 \pi$ for $\mathrm{VO}_{2}$ /sapphire at $\lambda \sim 11.75 \mu \mathrm{m}$ ) and substantial absorption as light propagates through the lossy film. As a result of these high losses, all of the incident light is absorbed after only a few passes through the thin film; $R=|r|^{2}$ already reaches $\sim 0.0006$ after including just the $\mathrm{m}=\{0,1,2,3\}$ terms in Eq. (1).

Critical coupling to a cavity consisting of an ultra-thin absorptive film, a dielectric spacer, and a quarter-wave stack reflector has been previously shown, ${ }^{7}$ but to our knowledge it has not been demonstrated for a resonator formed by an ultra-thin absorbing layer directly on an opaque substrate. We additionally note that a distinct but related condition for maximizing absorption has been recently theoretically proposed for a vanishingly thin film with $n \approx k$ embedded between two lossless dielectrics. ${ }^{28-30}$

Thermally tuning the phase co-existence in $\mathrm{VO}_{2}$ is equivalent to tracing out a path in $n-k$ space; this trajectory is plotted as a function of temperature in Fig. 3 (black curve) using the data from Ref. 17. We observe that at $\mathrm{T} \sim 342 \mathrm{~K}$, the index of $\mathrm{VO}_{2}$ passes almost exactly through the point of low reflectivity, confirming our experimental data. The phase transition in $\mathrm{VO}_{2}$ results in a very large change in its optical properties, enabling a change in device reflectivity on the order of unity.

Calculations show that the spectral position of the absorption maximum can be tailored over a significant portion of the $8 \mu \mathrm{m}-14 \mu \mathrm{m}$ atmospheric transparency window by changing the $\mathrm{VO}_{2}$ film thickness, making this geometry potentially useful for infrared detection and imaging applications. The calculated absorption maximum occurs at $\lambda \sim 10.5 \mu \mathrm{m}$ for a $20 \mathrm{~nm}$ $\mathrm{VO}_{2}$ film on sapphire, and can be continuously red-shifted with increasing $\mathrm{VO}_{2}$ thickness; for example, at $h=400 \mathrm{~nm}$, the dip is predicted to be at $\lambda \sim 13.3 \mu \mathrm{m}$ with $\mathrm{R} \sim 0.09$ (see supplementary material ${ }^{39}$ for experimental data on a sample with $h=80 \mathrm{~nm}$ ).

$\mathrm{VO}_{2}$ has long been investigated for applications in bolometers using the IMT, ${ }^{31}$ though this approach is not very practical due to the presence of hysteresis. ${ }^{32}$ Nonetheless, various vanadium oxides (usually mixed valence $\mathrm{VO}_{\mathrm{x}}$ ) are widely used for commercial bolometers at temperatures away from the IMT (e.g., Ref. 33). Recently, however, a bolometer based on non-hysteretic behavior within the transition region has been proposed, ${ }^{32}$ which may increase the temperature coefficient of resistance, leading to enhanced detectivity. Combining this approach with the perfect absorber geometry demonstrated in the present work could result in significantly improved infrared detection and imaging, especially if a dopant is used to decrease the transition temperature of $\mathrm{VO}_{2} \cdot{ }^{27}$

In order to extend the absorber concept demonstrated here to other spectral ranges, alternate materials for either the substrate or the ultra-thin film can be used. $\mathrm{VO}_{2}$ films, for example, can be grown on a number of substrates such as glass, silicon, germanium, titanium oxide, and indium-tin-oxide (ITO). ${ }^{19,34}$ In general, tunable highly absorptive layers can be created using a variety of correlated oxides, which exhibit phase co-existence in the vicinity of phase transitions ${ }^{19}$ as well as other phase change materials. ${ }^{35}$

In conclusion, we have demonstrated an absorber at $\lambda \sim 11.6 \mu \mathrm{m}$ comprising an ultra-thin $(\sim \lambda / 65)$ film of $\mathrm{VO}_{2}$ on a sapphire substrate that can be tuned into the perfect absorption state (with measured reflectivity of $\sim 0.0025$ ) by varying the temperature in proximity to the IMT. In this transitional state, the film comprises nanoscale structures of metallic- and insulator-state $\mathrm{VO}_{2}$, and the resulting effective medium behaves as a tunable disordered metamaterial with large optical absorption at infrared frequencies. The interaction of light with the $\mathrm{VO}_{2}$ and the underlying sapphire substrate results in essentially complete absorption of the incident light as a result of critical coupling. This effect involves very few optical round trips through the absorbing film with thickness much smaller than the wavelength. The absorber was brought into and out of its highly absorbing state by modification of the phase co-existence within the $\mathrm{VO}_{2}$ layer, achieving an on/off ratio of more than 300 in reflectivity. The absorber demonstrated here requires no nanofabrication steps beyond deposition of the $\mathrm{VO}_{2}$ film, and thus can easily be made to cover a large area. The use of the transitional state in a correlated oxide enables its use as a reconfigurable component of a wide variety of optical and optoelectronic systems such as bolometers, modulators, and tunable thermal emitters (thermal emission is closely linked to absorption via Kirchhoff's law ${ }^{36-38}$ ). The IMT in $\mathrm{VO}_{2}$ is known to occur on a picosecond timescale and can be triggered thermally, optically, or electrically, ${ }^{19}$ making $\mathrm{VO}_{2}$-based components promising for future optoelectronic devices.

The authors acknowledge helpful discussion with Y. Zhou, J. Fan, and N. Yu. This work was supported in part by the Defense Advanced Research Projects Agency (DARPA) N/MEMS S\&T Fundamentals program under Grant No. N66001-10-1-4008 issued by the Space and Naval Warfare Systems Center Pacific (SPAWAR). M. Kats is supported by the National Science Foundation through a Graduate Research Fellowship. J. Lin acknowledges the fellowship support from the Agency for Science, Technology and Research (A*STAR), Singapore. Financial support from ONR through Grant No. N00014-10-1-0131 is acknowledged. M. Qazilbash gratefully acknowledges financial support from the Jeffress Memorial Trust. D. Basov acknowledges financial support from AFOSR. M. Kats thanks K. Nadtochiy for the cover artwork.

${ }^{1}$ H. A. Haus, Waves and Fields in Optoelectronics (Prentice-Hall, New Jersey, 1984).

${ }^{2}$ R. H. Yan, R. J. Simes, and L. A. Coldren, IEEE Photon. Technol. Lett. 1, 273 (1989).

${ }^{3}$ A. Chin and T. Y. Chang, J. Vac. Sci. Technol. B. 8, 339 (1990).

${ }^{4}$ K. Kishino, M. S. Unlu, J.-I. Chyi, J. Reed, L. Arsenault, and H. Morkoc, IEEE J. Quantum Electron. 27, 2025 (1991).

${ }^{5}$ R. H. Yan, R. J. Simes, and L. A. Coldren, IEEE J. Quantum Electron. 27, 1922 (1991).

${ }^{6}$ K. K. Law, R. H. Yan, L. A. Coldren, and J. L. Merz, Appl. Phys. Lett. 57, 1345 (1990).

${ }^{7}$ J. R. Tischler, M. S. Bradley, and V. Bulovic, Opt. Lett. 31, 2045 (2006).

${ }^{8}$ Y. D. Chong, L. Ge, H. Cao, and A. D. Stone, Phys. Rev. Lett. 105, 053901 (2010). 
${ }^{9}$ W. Wan, Y. D. Chong, L. Ge, H. Noh, A. D. Stone, and H. Cao, Science 331, 889 (2011).

${ }^{10}$ H. Noh, Y. Chong, A. D. Stone, and H. Cao, Phys. Rev. Lett. 108, 186805 (2012).

${ }^{11}$ N. I. Landy, S. Sajuyigbe, J. J. Mock, D. R. Smith, and W. J. Padilla, Phys. Rev. Lett. 100, 207402 (2008).

${ }^{12}$ H. Tao, C. M. Bingham, A. C. Strikwerda, D. Pilon, D. Shrekenhamer, N. I. Landy, K. Fan, X. Zhang, W. J. Padilla, and R. D. Averitt, Phys. Rev. B 78, 241103(R) (2008).

${ }^{13}$ J. Hao, J. Wang, X. Liu, W. J. Padilla, L. Zhou, and M. Qiu, Appl. Phys. Lett. 96, 251104 (2010).

${ }^{14}$ C. Wu, B. Neuner III, and G. Shvets, Phys. Rev. B 84, 075102 (2011).

${ }^{15}$ N. Liu, M. Mesch, T. Weiss, M. Hentschel, and H. Giessen, Nano Lett. 10, 2342 (2010).

${ }^{16} \mathrm{M}$. Born and E. Wolf, Principles of Optics, 7 th ed. (Cambridge University Press, 2003).

${ }^{17}$ M. M. Qazilbash, M. Brehm, B.-G. Chae, P.-C. Ho, G. O. Andreev, B.-J. Kim, S. J. Yun, A. V. Balatsky, M. B. Maple, F. Keilmann, H.-T. Kim, and D. N. Basov, Science 318, 1750 (2007).

${ }^{18}$ H. S. Choi, J. S. Ahn, J. H. Jung, T. W. Noh, and D. H. Kim, Phys. Rev. B 54, 4621 (1996).

${ }^{19}$ Z. Yang, C. Ko, and S. Ramanathan, Annu. Rev. Mater. Res. 41, 337 (2011).

${ }^{20}$ W. R. Roach and I. Balberg, Solid State Commun. 9, 551 (1971).

${ }^{21}$ D. Ruzmetov, K. T. Zawilski, S. D. Senanayake, V. Narayanamurti, and S. Ramanathan, J. Phys.: Condens. Matter 20, 465204 (2008).

${ }^{22}$ T. Driscoll, H.-T. Kim, B.-G. Chae, B.-J. Kim, Y.-W. Lee, N. M. Jokerst, S. Palit, D. R. Smith, M. D. Ventra, and D. N. Basov, Science 325, 1518 (2009).

${ }^{23}$ M. J. Dicken, K. Aydin, I. M. Pryce, L. A. Sweatlock, E. M. Boyd, S. Walavalker, J. Ma, and H. A. Atwater, Opt. Express 17, 18330 (2009).
${ }^{24}$ M. Seo, J. Kyoung, H. Park, S. Koo, H. Kim, H. Bernien, B. J. Kim, J. H. Choe, Y. H. Ahn, H.-T. Kim, N. Park, Q.-H. Park, K. Ahn, and D. Kim, Nano Lett. 10, 2064 (2010).

${ }^{25}$ F. Gervais and B. Piriou, J. Phys. C: Solid State Phys. 7, 2374 (1974).

${ }^{26}$ E. D. Palik, Handbook of Optical Constants of Solids II (Elsevier Science, 1998).

${ }^{27}$ J. Nag and R. F. Haglund, J. Phys.: Condens. Matter 20, 264016 (2008).

${ }^{28}$ H. Bosman, Y. Y. Lau, and R. M. Gilgenbach, Appl. Phys. Lett. 82, 1353 (2003).

${ }^{29}$ C. Hagglund, S. P. Apell, and B. Kasemo, Nano Lett. 10, 3135 (2010).

${ }^{30}$ M. Pu, Q. Feng, M. Wang, C. Hu, C. Huang, X. Ma, Z. Zhao, C. Wang, and X. Luo, Opt. Express 20, 2246 (2012).

${ }^{31}$ R. S. Scott and G. E. Fredericks, Infrared Phys. 16, 619 (1976).

${ }^{32}$ M. Gurvitch, S. Luryi, A. Polyakov, and A. Shabalov, J. Appl. Phys. 106, 104504 (2009).

${ }^{33}$ C. Chen, X. Yi, X. Zhao, and B. Xiong, Sens. Actuators, A: Phys. 90, 212 (2001).

${ }^{34}$ H. Miyazaki and I. Yasui, J. Phys. D 39, 2220 (2006).

${ }^{35}$ M. Wuttig and N. Yamada, Nature Mater. 6, 824 (2007).

${ }^{36}$ N. J. Harrick and A. F. Turner, Appl. Opt. 9, 2111 (1970).

${ }^{37}$ M. Diem, T. Koschny, and C. M. Soukoulis, Phys. Rev. B 79, 033101 (2009).

${ }^{38}$ J. A. Mason, S. Smith, and D. Wasserman, Appl. Phys. Lett. 98, 241105 (2011).

${ }^{39}$ See the supplementary material at http://dx.doi.org/10.1063/1.4767646 for details on the sample preparation and optical measurements, as well as some additional calculated and experimental optical spectra.

${ }^{40}$ M. A. Kats, R. Blanchard, P. Genevet, and F. Capasso, "Nanometre optical coatings based on strong interference effects in highly absorbing media," Nature Mater. (published online). 